\title{
Reactive scattering with row-orthonormal hyperspherical coordinates. 4. Four-dimensional-space Wigner rotation function for pentaatomic systems
}

\author{
Aron Kuppermann \\ Received 18th December 2010, Accepted 14th February 2011 \\ DOI: $10.1039 / \mathbf{c 0 c p 0 2 9 0 7 f}$
}

The row-orthonormal hyperspherical coordinate (ROHC) approach to calculating state-to-state reaction cross sections and bound state levels of $N$-atom systems requires the use of angular momentum tensors and Wigner rotation functions in a space of dimension $N-1$. The properties of those tensors and functions are discussed for arbitrary $N$ and determined for $N=5$ in terms of the 6 Euler angles involved in 4-dimensional space.

\section{Introduction}

Accurate $a b$ initio calculations of state-to-state differential and integral cross sections of simple chemical reactions constitute an important and active field. The methods used include the solution of both the time-dependent and the time-independent Schrödinger equation, and are described in several excellent reviews ${ }^{1-5}$ and references therein. In addition, some of the characteristics of these methods have been recently compared. ${ }^{6}$ Such cross section calculations and the associated wave functions give important insight into the detailed mechanism of those reactions at the molecular level. Furthermore, they furnish benchmarks against which approximate methods may be tested. The latter must be used to extend these kinds of studies to larger systems for which accurate $a b$ initio calculations are not currently feasible.

In addition to Cartesian coordinates, hyperspherical coordinates of different varieties have been used to describe reactive scattering processes, ${ }^{7-25}$ as well as bound states. ${ }^{26,27}$ The row-orthonormal hyperspherical coordinate (ROHC) variety $^{28-34}$ has the useful property that the resulting many individual terms of a polyatomic system's Hamiltonian are invariant under a change of arrangement channel coordinates $^{30-34}$ (i.e., kinematic rotations), which makes them particularly appropriate for the study of chemical reactions. The formalism involved, for an $\mathrm{N}$-atom system, introduces the concepts of rotations and angular momenta in spaces of dimension $n=N-1$. As a result, such angular momenta and the associated Euler angles and Wigner rotation functions play an important role in the ROHC methodology, and are of central interest in this paper, which is organized as follows.

Arthur Amos Noyes Laboratory of Chemical Physics,

Division of Chemistry and Chemical Engineering,

California Institute of Technology, Pasadena, California 91125, USA.

E-mail:aron@caltech.edu
In Section 2 the definition and properties of ROHC are summarized, and in Section 3 coordinate rotations and Euler angles in $(n \geq 3)$-dimensional space are discussed. In Section 4 the tensor angular momentum in such space is analyzed and in Section 5 the corresponding Wigner rotation operator and function are investigated. A summary and conclusions are given in Section 6.

\section{Background}

The definition and properties of the ROHC for $N$-atom systems for $N \geq 3$ have been given previously ${ }^{29-34}$ and will only be summarized below succinctly. Let the corresponding set of $N-1 \lambda$-arrangement mass-scaled Jacobi vectors be $\mathbf{r}_{\lambda}^{(1)}, \mathbf{r}_{\lambda}^{(2)}, \ldots, \mathbf{r}_{\lambda}^{(N-1)}$, and $\mu$ its reduced mass. The associated space-fixed Jacobi matrix $\rho_{\lambda}^{s f}$ is defined by

$$
\begin{aligned}
\rho_{\lambda}^{s f} & =\left(\mathbf{r}_{\lambda}^{(1)} \mathbf{r}_{\lambda}^{(2)} \ldots \mathbf{r}_{\lambda}^{(N-1)}\right) \\
& =\left(\begin{array}{llll}
x_{\lambda_{1}}^{(1)} & x_{\lambda_{1}}^{(2)} & \ldots & x_{\lambda_{1}}^{(n-1)} \\
x_{\lambda_{2}}^{(1)} & x_{\lambda_{2}}^{(2)} & \ldots & x_{\lambda_{2}}^{(n-1)} \\
x_{\lambda_{3}}^{(1)} & x_{\lambda_{3}}^{(2)} & \ldots & x_{\lambda_{3}}^{(n-1)}
\end{array}\right)
\end{aligned}
$$

where $x_{\lambda_{1}}^{(j)} \equiv x_{\lambda}^{(j)}, x_{\lambda_{2}}^{(j)} \equiv y_{\lambda}^{(j)}, x_{\lambda_{3}}^{(j)} \equiv z_{\lambda}^{(j)}$ are the Cartesian spacefixed components of $\mathbf{r}_{\lambda_{j}}(j=1,2, \ldots, N-1)$. As a result of the singular value theorem for real matrices, ${ }^{35,36} \rho_{\lambda}^{s f}$ can, for $N \geq 4$, be put in the form ${ }^{2,8,33,37-39}$

$$
\rho_{\lambda}^{s f}=(-1)^{\chi} \mathbf{R}^{(3)}\left(\mathbf{a}_{\lambda}\right) \rho \mathbf{N}(\theta, \phi) \mathbf{P} \tilde{\mathbf{R}}^{(N-1)}\left(\boldsymbol{\delta}_{\lambda}\right)
$$

where $x_{\lambda}$ is a chirality coordinate that can assume the values 0 or $1, \mathbf{a}_{\lambda} \equiv\left(a_{\lambda}, b_{\lambda}, c_{\lambda}\right)$ are the 3 Euler angles that rotate the spacefixed frame $G x y z$ (centered on the system's center of mass $G$ ) to the principal-axes-of-inertia body-fixed frame $G x_{1}^{I \lambda} x_{2}^{I \lambda} x_{3}^{I \lambda} \equiv$ $G x^{I \lambda} y^{I \lambda} z^{I \lambda}$ and $\tilde{\mathbf{R}}^{(3)}\left(\mathbf{a}_{\lambda}\right)$ is the transpose of the corresponding proper coordinate rotation matrix $\mathbf{R}^{(3)}\left(\mathbf{a}_{\lambda}\right)$. (The expression "coordinate" rotation matrix is used to distinguish it from the 
"Wigner" rotation matrix considered at the end of this section.) This frame is not arbitrary and is required for the validity of that theorem. Furthermore, $\mathbf{N}(\theta, \phi)$ is the $3 \times 3$ diagonal matrix.

$$
\mathbf{N}(\theta, \phi)=\left(\begin{array}{ccc}
\sin \theta \cos \phi & 0 & 0 \\
0 & \sin \theta \sin \phi & 0 \\
0 & 0 & \cos \theta
\end{array}\right)
$$

where $\theta$ and $\phi$ are hyperangles closely related to the system's principal moments of inertia, ${ }^{33}$ and $\rho \geq 0$ is the system's $\lambda$-independent hyperradius defined by

$$
\rho^{2}=\sum_{j=1}^{N-1}\left(x_{\lambda}^{(j)^{2}}+y_{\lambda}^{(j)^{2}}+z_{\lambda}^{(j)^{2}}\right)
$$

In addition, $\mathbf{R}^{(N-1)}\left(\boldsymbol{\delta}_{\lambda}\right)$ is the $(N-1) \times(N-1)$ proper orthogonal matrix that rotates a set of $N-1$ Cartesian axes $\mathrm{OX}_{1} \mathrm{X}_{2} \ldots \mathrm{X}_{N-1}$ in an $(N-1)$-dimensional mathematical space, via the $\sigma=N(N-1) / 2$ Euler angles $\boldsymbol{\delta}_{\lambda} \equiv\left(\delta_{\lambda}^{(1)}, \delta_{\lambda}^{(1)}, \ldots, \delta_{\lambda}^{(\sigma)}\right)$, to the set of $N-1$ Cartesian axes $\mathrm{OX}_{\lambda_{1}} \mathrm{X}_{\lambda_{2}} \ldots \mathrm{X}_{\lambda_{N-1}}$ in that same space, as discussed in greater detail in Section 3. Finally $\mathbf{P}$ is the $3 \times(N-1)$ matrix $^{40}$

$$
\mathbf{P}=\left(\begin{array}{cccccc}
1 & 0 & 0 & 0 & \ldots & 0 \\
0 & 1 & 0 & 0 & \ldots & 0 \\
0 & 0 & 1 & 0 & \ldots & 0
\end{array}\right)=\left(\mathbf{I}^{(3)} \mathbf{0}_{3 \times(N-4)}\right)
$$

where $\mathbf{I}^{(3)}$ and $\mathbf{0}_{3 \times(N-4)}$ are, respectively, the $3 \times 3$ identity matrix and the $3 \times(N-4)$ null matrix. As a result, $\mathbf{P} \tilde{\mathbf{R}}^{N-1}\left(\boldsymbol{\delta}_{\lambda}\right)$ is a $3 \times(N-1)$ row-orthonormal matrix, formed by the first 3 rows of $\tilde{\mathbf{R}}^{(N-1)}$, which are normalized and orthogonal to each other. It should be noted that the number of $\delta_{\lambda}^{(p)}$ in $\mathbf{P} \tilde{\mathbf{R}}^{(N-1)}$ is $\nu=3 N-9$, which is smaller than $\sigma$ for $N>5$ because of the smaller number of elements in that matrix as compared to $\tilde{\mathbf{R}}^{(N-1)}$ for such values of $N$. This matter is further discussed in Section 3.3.

As a result of the presence of the $(N-1) \times(N-1)$ orthogonal matrix $\tilde{\mathbf{R}}^{(N-1)}\left(\boldsymbol{\delta}_{\lambda}\right)$ in $(2.2)$, rotations and the associated angular momenta in $(N-1)$-dimensional space appears in the ROHC formulation of reactive scattering of $N$-atom systems. As mentioned in Section 1, the many terms in the Hamiltonian of these systems are kinematic-rotation invariant, which makes them particularly useful for the description of reactive processes. In order to use these coordinates for state-to-state reaction cross section calculations, it becomes important to derive expressions for the corresponding Wigner rotation function. ${ }^{41}$ For tetraatomic systems, $N-1=3$ and no new theory is necessary. However, for $N \geq 5$, the spaces involved have dimensions of 4 or greater. In the present paper we pay particular attention to pentaatomic systems, and develop the theory of the Wigner rotation function in 4-dimensional space, for which the number of Euler angles is $\sigma=6$.

\section{Rotations and Euler angles in ( $n \geq 3$ )-dimensional space}

Since rotations in 3-dimensional space involve 3 Euler angles, it might naïvely be thought that for $n$-dimensional space, $n$ Euler angles are involved. However, a more careful analysis leads to a different conclusion. For the present paper, $n=N-1$.

Let the coordinates of a general point $\mathrm{P}$ in the $n$-dimensional space frame $\mathrm{OX}_{1}^{(n)} \mathrm{X}_{2}^{(n)} \ldots \mathrm{X}_{n}^{(n)}$ be $\left(\mathrm{X}_{1}^{(n)}, \mathrm{X}_{2}^{(n)}, \ldots, \mathrm{X}_{n}^{(n)}\right)$. A proper or improper rotation of these axes to $\mathrm{O} \overline{\mathrm{X}}_{1}^{(n)} \overline{\mathrm{X}}_{2}^{(n)} \ldots \overline{\mathrm{X}}_{n}^{(n)}$ is defined by

$$
\overline{\mathbf{X}}^{(n)}=\mathbf{R}^{(n)} \mathbf{X}^{(n)}
$$

where $\mathbf{X}^{(n)}$ (or $\overline{\mathbf{X}}^{(n)}$ ) is the $n$-dimensional column vector whose elements are the $\mathrm{X}_{j}^{(n)}$ (or $\left.\overline{\mathrm{X}}_{j}^{(n)}\right), j=1,2, \ldots, n$ and $\mathbf{R}^{(n)}$ is an $n$-dimensional orthogonal square matrix whose determinant is $+1(-1)$ for proper (improper) rotations.

\subsection{The primary rotations}

The $n^{2}$ elements of the orthogonal matrix $\mathbf{R}^{(n)}$ of (3.1) satisfy $n$ row-normalization conditions as well as $(1 / 2) n(n-1)$ roworthogonality conditions and therefore the number of degrees of freedom of this matrix is

$$
\sigma=n^{2}-n-(1 / 2) n(n-1)=(1 / 2) n(n-1)=\left(\begin{array}{l}
n \\
2
\end{array}\right)
$$

As a result, a general rotation of $\mathrm{OX}_{1}^{(n)} \mathrm{X}_{2}^{(n)} \ldots \mathrm{X}_{n}^{(n)}$ can be represented by a sequence of rotations of pairs of axes (or of planes) $\mathrm{OX}_{i}^{(n)} \mathrm{X}_{j}^{(n)} \rightarrow \mathrm{OX}_{i}^{(n)} \mathrm{OX}_{j}^{(n)^{\prime}}$, since the number of such pairs (or of planes) is also $\sigma$. Except for $n=3$, for which $\sigma=3$, we have $\sigma>n$, i.e., the number of distinct $\mathrm{OX}_{i}^{(n)} \mathrm{X}_{j}^{(n)}$ planes exceeds the dimensionality of the space. The positive sense of these rotations is specified by requiring that $\mathrm{OX}_{i}^{(n)}$ should move towards $\mathrm{OX}_{j}^{(n)}$. The remaining axes $\mathrm{OX}_{k}^{(n)}$, with $k \neq i, j$, are maintained fixed under each such rotation and are therefore the axes around which that rotation takes place. We call these rotations "primary". The coordinates $\mathbf{X}^{(n)}$ of a point $\mathbf{P}$ are required to transform under such rotations to $\mathbf{X}^{(n) \prime}$ according to

$$
\begin{aligned}
& \mathrm{X}_{i}^{(n)^{\prime}}=\cos \omega \mathrm{X}_{i}^{(n)}+\sin \omega \mathrm{X}_{j}^{(n)} \\
& \mathrm{X}_{j}^{(n)^{\prime}}=-\sin \omega \mathrm{X}_{i}^{(n)}+\cos \omega \mathrm{X}_{j}^{(n)} \quad i, j=1,2, \ldots, n i \neq j \\
& \mathrm{X}_{k}^{(n)^{\prime}}=\mathrm{X}_{k}^{(n)} \quad \text { for } k \neq i, j
\end{aligned}
$$

where $\omega$ is the rotation angle. This corresponds to a proper rotation described by an $n \times n$ proper orthogonal matrix labelled $\mathbf{R}_{i j}^{(n)}(\omega)$, called a primary coordinate rotation matrix. Consider the permutation of the indices $1,2, \ldots, n$ that places $i$ in first place, $j$ in second place and leaves the ordering of the remaining indices unchanged. We require that all rotations defined by (3.3) have the same "handedness" by making this permutation even. For example, for $n=4$, we allow the rotation $\mathrm{OX}_{3}^{(4)} \mathrm{X}_{1}^{(4)} \rightarrow \mathrm{OX}_{3}^{(4)^{\prime}} \mathrm{X}_{1}^{(6)^{\prime}}$ because the permutation $(3,1,2,4)$ is even, but we do not allow the rotation $\mathrm{OX}_{1}^{(4)} \mathrm{X}_{3}^{(4)} \rightarrow \mathrm{OX}_{1}^{(4)^{\prime}} \mathrm{X}_{3}^{(4)^{\prime}}$ because the permutation $(1,3,2,4)$ is odd. This rule is fulfilled for the usual 3-dimensional axes rotations. Adopting such a requirement, the number of primary rotations is equal to the number of distinct $\mathrm{OX}_{i}^{(n)} \mathrm{X}_{j}^{(n)}$ pairs of axes (or planes). 
(a)



(b)

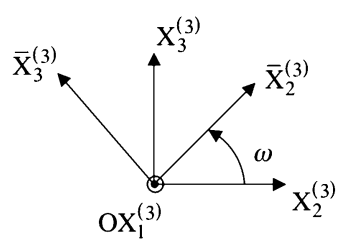

(c)

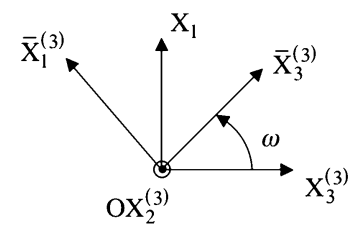

Fig. 1 Allowed rotations for of pairs of axes (or of planes) of the OX ${ }_{1}^{(3)} \mathrm{X}_{2}^{(3)} \mathrm{X}_{3}^{(3)}$ frame in a 3-dimensional space. (a) The axes $\mathrm{OX}_{1}^{(3)} \mathrm{X}_{2}^{(3)}$ are rotated by $\omega$ to $O \bar{X}_{1}^{(3)} \bar{X}_{2}^{(3)}$ with $\mathrm{OX}_{3}^{(3)}$ fixed and perpendicular to the plane of the Figure, pointing upwards, as indicated by the circle at the origin; (b) and (c) are similar, with the indices 1,2,3 permuted cyclically to $2,3,1$ and $3,1,2$ respectively.

Examples of primary coordinate rotation matrices are:

(1) For $n=3$

$$
\begin{aligned}
\mathbf{R}_{12}^{(3)}(\omega) & =\left(\begin{array}{ccc}
\cos \omega & \sin \omega & 0 \\
-\sin \omega & \cos \omega & 0 \\
0 & 0 & 1
\end{array}\right) \\
\mathbf{R}_{23}^{(3)}(\omega) & =\left(\begin{array}{ccc}
1 & 0 & 0 \\
0 & \cos \omega & \sin \omega \\
0 & -\sin \omega & \cos \omega
\end{array}\right) \\
\mathbf{R}_{31}^{(3)}(\omega) & =\left(\begin{array}{ccc}
\cos \omega & 0 & -\sin \omega \\
0 & 1 & 0 \\
\sin \omega & 0 & \cos \omega
\end{array}\right)
\end{aligned}
$$

For $\omega>0, \mathbf{R}_{12}^{(3)}$ rotates $\mathrm{OX}_{1}^{(3)} \mathrm{X}_{2}^{(3)}$ (in the sense $\mathrm{OX}_{1}^{(3)} \rightarrow \mathrm{OX}_{2}^{(3)}$ ) to $\mathrm{OX} \bar{X}_{1}^{(3)} \overline{\mathrm{X}}_{2}^{(3)}$ by $\omega$ with $\mathrm{OX}_{3}^{(3)}$ fixed, $\mathbf{R}_{23}^{(3)}$ rotates $\mathrm{OX}_{2}^{(3)} \mathrm{X}_{3}^{(3)}$ to $\mathrm{OX} \bar{X}_{2}^{(3)} \overline{\mathrm{X}}_{3}^{(3)}$ with $\mathrm{OX}_{1}^{(3)}$ fixed, and $\mathbf{R}_{31}^{(3)}$ rotates $\mathrm{OX}_{3}^{(3)} \mathrm{X}_{1}^{(3)}$ to $\mathrm{O} \overline{\mathrm{X}}_{3}^{(3)} \overline{\mathrm{X}}_{1}^{(3)}$ with $\mathrm{OX} \overline{\mathrm{X}}_{2}^{(3)}$ fixed. These rotations are depicted in Fig. 1.

(2) For $n=4$

$$
\begin{aligned}
\mathbf{R}_{12}^{(4)}(\omega) & =\left(\begin{array}{cccc}
\cos \omega & \sin \omega & 0 & 0 \\
-\sin \omega & \cos \omega & 0 & 0 \\
0 & 0 & 1 & 0 \\
0 & 0 & 0 & 1
\end{array}\right) \\
\mathbf{R}_{23}^{(4)}(\omega) & =\left(\begin{array}{cccc}
1 & 0 & 0 & 0 \\
0 & \cos \omega & \sin \omega & 0 \\
0 & -\sin \omega & \cos \omega & 0 \\
0 & 0 & 0 & 1
\end{array}\right)
\end{aligned}
$$

$$
\begin{aligned}
\mathbf{R}_{14}^{(4)}(\omega) & =\left(\begin{array}{cccc}
\cos \omega & 0 & 0 & \sin \omega \\
0 & 1 & 0 & 0 \\
0 & 0 & 1 & 0 \\
-\sin \omega & 0 & 0 & \cos \omega
\end{array}\right) \\
\mathbf{R}_{42}^{(4)}(\omega) & =\left(\begin{array}{ccccc}
1 & 0 & 0 & 0 \\
0 & \cos \omega & 0 & -\sin \omega \\
0 & 0 & 1 & 0 \\
0 & \sin \omega & 0 & \cos \omega
\end{array}\right) \\
\mathbf{R}_{31}^{(4)}(\omega) & =\left(\begin{array}{ccccc}
\cos \omega & 0 & -\sin \omega & 0 \\
0 & 1 & 0 & 0 \\
\sin \omega & 0 & \cos \omega & 0 \\
0 & 0 & 0 & 1
\end{array}\right) \\
\mathbf{R}_{34}^{(4)}(\omega) & =\left(\begin{array}{ccccc}
1 & 0 & 0 & 0 \\
0 & 1 & 0 & 0 \\
0 & 0 & \cos \omega & \sin \omega \\
0 & 0 & -\sin \omega & \cos \omega
\end{array}\right)
\end{aligned}
$$

In each of these matrices, the diagonal elements equal to 1 indicate the axes that are fixed, i.e., the rotation axes.

\subsection{The coordinate rotation matrix and the Euler angles}

The proper coordinate rotation matrix $\mathbf{R}^{(n)}$ is expressed as a product of $\sigma$ primary coordinate rotation matrices $\mathbf{R}_{i j}^{(n)}(\omega)$ chosen from the allowed set of such matrices. For example, for the usual $n=3$ case, $\sigma$ is equal to 3 and we chose the corresponding 3 matrices from the set of (3.4). Although we could choose all of them to have different pairs of $i, j$ subscripts, it is more convenient to make two of those pairs the same but associate them with different rotation angles. The usual $\mathbf{R}^{(3)}$ is

$$
\begin{aligned}
\mathbf{R}^{(3)}\left(\delta^{(1)}, \delta^{(2)}, \delta^{(3)}\right)= & \mathbf{R}_{12}^{(3)}\left(\delta^{(3)}\right) \mathbf{R}_{31}^{(3)}\left(\delta^{(2)}\right) \mathbf{R}_{12}^{(3)}\left(\delta^{(1)}\right) \\
= & \left(\begin{array}{ccc}
\cos \delta^{(3)} & \sin \delta^{(3)} & 0 \\
-\sin \delta^{(3)} & \cos \delta^{(3)} & 0 \\
0 & 0 & 1
\end{array}\right) \\
& \times\left(\begin{array}{cccc}
\cos \delta^{(2)} & 0 & -\sin \delta^{(2)} \\
0 & 1 & 0 \\
\sin \delta^{(2)} & 0 & \cos \delta^{(2)}
\end{array}\right) \\
& \times\left(\begin{array}{ccc}
\cos \delta^{(1)} & \sin \delta^{(1)} & 0 \\
-\sin \delta^{(1)} & \cos \delta^{(1)} & 0 \\
0 & 0 & 1
\end{array}\right)
\end{aligned}
$$

in which $\mathbf{R}_{23}^{(3)}$, which describes a primary rotation for which the $\mathrm{OX}_{1}$ axis is unchanged, is not included. Instead $\mathbf{R}_{12}^{(3)}$, 
corresponding to a primary rotation in which the $\mathrm{OX}_{3}$ axis is unchanged, is used twice. The reason is that the corresponding components of the angular momentum tensors $\hat{\mathbf{L}}^{(3)}$ and $\overline{\mathbf{L}}^{(3)}$, namely $\hat{\mathrm{L}}_{12}^{(3)}=(\hbar / i)\left(\partial / \delta^{(1)}\right)$ and $\hat{\mathrm{L}}_{12}^{3^{\prime \prime}}=(\hbar / i)\left(\partial / \delta^{(3)}\right)$, are particularly simple compared to the other components (see Section 4.3) and greatly simplify the associated angular momentum algebra.

Similarly, for $n=4$, we chose the following expression for $\mathbf{R}^{(4)}(\boldsymbol{\delta})$, with $\boldsymbol{\delta}=\left(\delta^{(1)}, \delta^{(2)}, \ldots, \delta^{(6)}\right)$ :

$$
\mathbf{R}^{(4)}(\boldsymbol{\delta})=\mathbf{R}_{14}^{(4)}\left(\delta^{(6)}\right) \mathbf{R}_{42}^{(4)}\left(\delta^{(5)}\right) \mathbf{R}_{34}^{(4)}\left(\delta^{(4)}\right) \mathbf{R}_{12}^{(4)}\left(\delta^{(3)}\right) \mathbf{R}_{31}^{(4)}\left(\delta^{(2)}\right) \mathbf{R}_{12}^{(4)}\left(\delta^{(1)}\right)
$$

The last 3 matrices are closely related to the corresponding $\mathbf{R}_{12}^{(3)}\left(\delta^{(1)}\right), \mathbf{R}_{31}^{(3)}\left(\delta^{(2)}\right)$ and $\mathbf{R}_{12}^{(3)}\left(\delta^{(3)}\right)$ matrices, having an extra row and column of zeros, except for the $(4,4)$ element, which is 1 . This choice is convenient for relating the $n=4$ to the $n=3$ angular momenta. Again, in (3.7) the matrix $\mathbf{R}_{23}^{(4)}$ is not included, in order to permit the appearance of $\mathbf{R}_{12}^{(4)}$ twice. In general, for an $(n \geq 3)$-dimensional space, the Euler angles are $\boldsymbol{\delta} \equiv\left(\delta^{(1)}, \delta^{(2)}, \ldots, \delta^{(\sigma)}\right)$ with $\sigma=(1 / 2) n(n-1)$. We choose the corresponding $\mathbf{R}^{(n)}(\boldsymbol{\delta})$ as the $n \times n$ proper orthogonal matrix

$$
\mathbf{R}^{(n)}(\boldsymbol{\delta})=\prod_{p=1}^{\sigma} \mathbf{R}_{i_{p j} j_{p}}^{(n)}\left(\delta^{(p)}\right)
$$

where the $\mathbf{R}_{i_{p} j_{p}}^{(n)}$ are selected from a set of allowed primary matrices and the order of the factors in eqn (3.8) is, from right to left, $p=1,2, \ldots, \sigma$.

The number $\sigma$ of Euler angles in $\boldsymbol{\delta}$ is equal (as indicated in Section 3.1) to the number of distinct $\mathrm{OX}_{i}^{(n)} \mathrm{X}_{j}^{(n)}$ planes. We impose on (3.8) the condition that there be a one-to-one correspondence between those angles and $\mathbf{R}^{(n)}$, i.e., no two sets of Euler angles should give the same $\mathbf{R}^{(n)}$ and vice versa. This requires that for two consecutive $\mathbf{R}_{i_{p} j_{p}}^{(n)}$ and $\mathbf{R}_{i_{p+1} j_{p+1}}^{(n)}$ matrices we should have $i_{p+1} \neq i_{p}$ and $j_{p+1} \neq j_{p}$, since otherwise any two pairs of angles $\delta^{(p)}$ and $\delta^{(p+1)}$ whose sum is the same will give the same $\mathbf{R}^{(n)}$.

A choice of $\mathbf{R}^{(n)}(\boldsymbol{\delta})$ for $n \geq 4$ that satisfies these constraints and is a generalization of (3.6) and (3.7) is:

$$
\begin{aligned}
& \mathbf{R}^{(n)}(\boldsymbol{\delta})=\mathbf{R}_{1 n}^{(n)}\left(\delta^{(\sigma)}\right) \mathbf{R}_{n 2}^{(n)}\left(\delta^{(\sigma-1)} \mathbf{R}_{3 n}^{(n)}\left(\delta^{(\sigma-2)}\right) \mathbf{R}_{n 4}^{(n)}\left(\delta^{(\sigma-3)}\right) \ldots\right. \\
& \mathbf{R}_{14}^{(n)}\left(\delta^{(6)}\right) \mathbf{R}_{42}^{(n)}\left(\delta^{(5)}\right) \mathbf{R}_{34}^{(n)}\left(\delta^{(4)}\right) \mathbf{R}_{12}^{(n)}\left(\delta^{(3)}\right) \mathbf{R}_{31}^{(n)}\left(\delta^{(2)}\right) \mathbf{R}_{12}{ }^{(n)}\left(\delta^{(1)}\right)
\end{aligned}
$$

In this expression, the last 6 matrices are obtained from the corresponding $\mathbf{R}_{i j}^{(4)}$ by adding $n-4$ rows and columns of zeros for the off-diagonal elements and setting the additional $n-4$ diagonal elements equal to 1 . In all other $\mathbf{R}_{i_{p} j_{p}}^{(n)}\left(\delta^{(p)}\right)$ we set

(a) Diagonal elements

$$
\left[\mathbf{R}_{i_{p} j_{p}}^{(n)}\left(\delta^{(p)}\right)\right]_{r r}= \begin{cases}\cos \delta^{(p)} & \text { for } r=i_{p}, j_{p} \\ 1 & \text { otherwise }\end{cases}
$$

(b) Off-diagonal elements

$$
\left[\mathbf{R}_{i_{p} j_{p}}^{(n)}\left(\delta^{(p)}\right)\right]_{r s}=\frac{i_{p}-j_{p}}{\left|i_{p}-j_{p}\right|} \begin{cases}(-1)^{r+s} \sin \delta^{(p)} & \text { for } r=i_{p}, s=j_{p} \\ -(-1)^{r+s} \sin \delta^{(p)} & \text { for } r=j_{p}, s=i_{p} \\ 0 & \text { otherwise }\end{cases}
$$

Expressions (3.10) and (3.11) yield matrices that generate axes rotations which satisfy the handedness conservation requirement imposed after (3.3).

For $n=4$ (3.9) is the same as (3.7). Expressions (3.10) and (3.11) are the generalizations of the $n=3$ case to arbitrary $n>3$.

\subsection{The number and ranges of the internal Euler angles}

Care must be taken in applying the properties of rotations in $n$-dimensional space to the ROHC theory of reactive scattering in polyatomic systems. In particular, the number and range of the Euler angles in $n$-dimensional space differ in general from the corresponding values in ROHC scattering theory.

a The number of internal Euler angles. As mentioned in Section 2, for an $N$-atom system there are $\nu=3 N-9$ internal Euler hyperangles $\delta^{(p)}(p=1,2, \ldots, \nu)$ in $\mathbf{P} \tilde{\mathbf{R}}^{(N-1)}$. However, the space in which the orthogonal matrix $\tilde{\mathbf{R}}^{(N-1)}$ of $(2.2)$ is defined is $(N-1)$-dimensional and is parameterized by $\sigma=$ $(1 / 2)(N-1)(N-2)$ rotational Euler angles $\delta_{\lambda}^{(p)}(p=1,2, \ldots, \sigma)$, as further discussed in Section 3.1. Whereas $\nu$ increases linearly with $N, \sigma$ increases quadratically. In general, $\nu \leq \sigma$. For $N=4, \nu=\sigma=3$ and for $N=5, \nu=\sigma=6$. For these two cases, $\nu$ and $\sigma$ are the same. However, for $N \geq 6, \nu<\sigma$ and there are fewer angles in $\mathbf{P} \tilde{\mathbf{R}}^{(N-1)}$ than in $\tilde{\mathbf{R}}^{(N-1)}$.

The reason why these two numbers of Euler angles are different for this case is as follows. For the full $(N-1)$-dimensional space the number of distinct primary rotations is $\sigma=\left(\begin{array}{c}N-1 \\ 2\end{array}\right)$. However, for the ROHC space, involving a $3 \times(N-1)$ row-orthogonal matrix, $\kappa=\left(\begin{array}{c}N-4 \\ 2\end{array}\right)$ of those rotations are not allowed, as they are associated with an $(N-4)$-dimensional sub-space whose dimensionality has been decreased by 3 . It is easy to see that, for $(N \geq 6)$,

$$
\sigma-\kappa=\left(\begin{array}{c}
N-1 \\
2
\end{array}\right)-\left(\begin{array}{c}
N-4 \\
2
\end{array}\right)=3 N-9=\nu
$$

These $\kappa$ excluded rotations correspond to the $\kappa$ Euler angles $\delta_{\lambda}^{(p)}$ with $\nu<p \leq \sigma$ associated with the first $\kappa$ matrices $\mathbf{R}_{i_{p} j_{p}}^{(n)}\left(\delta_{\lambda}^{(p)}\right)$ (and the corresponding $\mathrm{OX}_{i_{p}} \mathrm{X}_{j_{p}}$ planes) in the r.h.s. of (3.9) for $n=N-1$. As a result, the matrix $\mathbf{P} \tilde{\mathbf{R}}^{(N-1)}\left(\boldsymbol{\delta}_{\lambda}\right)$ of (2.2) only contains the permitted ROHC Euler angles $\delta_{\lambda}^{(p)}$ with $p$ having the values $1,2, \ldots, \nu=3 N-9$.

b The ranges of the internal Euler angles. For tetraatomic systems, the ranges of the Euler angles in the associated 3-dimensional space are given, as usual, by

$$
0 \leq \delta_{\lambda}^{(1)}, \delta_{\lambda}^{(3)}<2 \pi \quad 0 \leq \delta_{\lambda}^{(2)} \leq \pi
$$

whereas the ranges of the corresponding ROHC hyperangles, also 3 in number, are given by ${ }^{30}$

$$
0 \leq \delta_{\lambda}^{(1)}, \delta_{\lambda}^{(3)}<\pi \quad 0 \leq \delta_{\lambda}^{(2)} \leq \pi
$$

The reason for this difference is that (3.13) results from the requirement that there be a 1 to 1 correspondence between Euler angles and rotations of axes in a three-dimensional space, whereas (3.14) is a consequence of requiring that there 
be a 1 to 1 correspondence between the 9 elements of the two sides of (2.2) for the $N=3$ case.

For pentaatomic systems the corresponding ranges are

$$
0 \leq \delta_{\lambda}^{(1)}, \delta_{\lambda}^{(3)}, \delta_{\lambda}^{(4)}<2 \pi \quad 0 \leq \delta_{\lambda}^{(2)}, \delta_{\lambda}^{(5)}, \delta_{\lambda}^{(6)} \leq \pi
$$

for rotations in 4-dimensional space and

$0 \leq \delta_{\lambda}^{(4)}<2 \pi \quad 0<\delta_{\lambda}^{(1)}, \delta_{\lambda}^{(2)}, \delta_{\lambda}^{(3)}, \delta_{\lambda}^{(5)}, \delta_{\lambda}^{(6)} \leq \pi$

for the corresponding ROHC. ${ }^{33}$

Similar reasoning leads to different ranges of corresponding $\delta_{\lambda}^{(p)}$ for all $N \geq 4$.

\section{Angular momentum tensors in ( $n \geq 3$ )-dimensional space}

\subsection{The angular momentum tensor operator}

The angular momentum operator of a point $\mathrm{P}$ in $n$-dimensional space in the frame defined at the beginning of Section 3 is a skew-symmetric tensor operator $\hat{\mathbf{L}}^{(n)}$ of order 2 whose $n^{2}$ elements are defined by

$$
\hat{\mathrm{L}}_{i j}^{(n)}=\frac{\hbar}{\mathrm{i}}\left(\mathrm{X}_{i}^{(n)} \frac{\partial}{\partial \mathrm{X}_{j}^{(n)}}-\mathrm{X}_{j}^{(n)} \frac{\partial}{\partial \mathrm{X}_{i}^{(n)}}\right) \quad i, j=1,2, \ldots, n
$$

It can be represented by a square-matrix operator of dimensions $n \times n$. Upon the rotation defined by (3.1), $\hat{\mathbf{L}}^{(n)}$ transforms to $\overline{\hat{\mathbf{L}}}^{(n)}$ according to the tensor relation

$$
\overline{\hat{\mathbf{L}}}^{(n)}=\mathbf{R}^{(n)} \hat{\mathbf{L}}^{(n)} \tilde{\mathbf{R}}^{(n)}
$$

The $\mathbf{R}^{(n)}$ can be chosen to be the function of the Euler angles $\boldsymbol{\delta}$ defined by (3.9). It should be stressed that $\hat{\mathrm{L}}$ acts on $\mathbf{X}^{(n)}$ but not on $\boldsymbol{\delta}$.

For 3-dimensional space, $\hat{\mathbf{L}}^{(3)}$ is given by

$$
\hat{\mathbf{L}}^{(3)}=\left(\begin{array}{ccc}
0 & \hat{\mathrm{L}}_{12}^{(3)} & -\hat{\mathrm{L}}_{31}^{(3)} \\
-\hat{\mathrm{L}}_{12}^{(3)} & 0 & \hat{\mathrm{L}}_{23}^{(3)} \\
\hat{\mathrm{L}}_{31}^{(3)} & -\hat{\mathbf{L}}_{23}^{(3)} & 0
\end{array}\right)
$$

If we choose $\mathbf{R}^{(3)}$ to be a proper orthogonal matrix, (4.2) is equivalent to

$$
\left(\begin{array}{c}
\overline{\hat{\mathbf{L}}}_{23}^{(3)} \\
\overline{\hat{\mathbf{L}}}_{31}^{(3)} \\
\overline{\hat{\mathbf{L}}}_{12}^{(3)}
\end{array}\right)=\mathbf{R}^{(3)}\left(\begin{array}{c}
\hat{\mathbf{L}}_{23}^{(3)} \\
\hat{\mathbf{L}}_{31}^{(3)} \\
\hat{\mathbf{L}}_{12}^{(3)}
\end{array}\right)
$$

i.e., 3 independent elements of $\hat{\mathbf{L}}^{(3)}$ can be assembled as a column that transforms under proper rotations as a vector. ${ }^{42}$ This is the usual angular momentum vector of 3-dimensional space in the $\mathrm{O} x y z$ frame with components $\hat{\mathrm{L}}_{x}=\hat{\mathrm{L}}_{23}^{(3)}, \hat{\mathrm{L}}_{y}=\hat{\mathrm{L}}_{31}^{(3)}$ and $\hat{\mathrm{L}}_{z}=\hat{\mathrm{L}}_{12}^{(3)}$. However, for $n>3$ the distinct elements of $\hat{\mathbf{L}}^{(n)}$ cannot be assembled as a column that transforms under such rotations as a vector, and its tensor nature is essential. As shown previously, ${ }^{33}$ (4.1) can be expressed in terms of a generalization of the Levi-Cevita density, which simplifies some aspects of the algebra of these angular momentum tensor operators, but this alternate form is not needed for the purposes of the present paper.

\subsection{Angular momentum for primary rotations in terms of the axes rotation angle}

Let us consider a primary rotation of the $\mathrm{OX}_{i}^{(n)} \mathrm{X}_{j}^{(n)}$ plane by an angle $\omega$, as described in Section 3.1. Let $g\left(\mathbf{X}^{(n)}\right)$ be an arbitrary function of $\mathbf{X}^{(n)}$ and let us consider a primary rotation of the $\mathrm{OX}_{i}^{(n)} \mathrm{X}_{j}^{(n)}$ plane to $\mathrm{O} \overline{\mathrm{X}}_{i}^{(n)} \overline{\mathrm{X}}_{j}^{(n)}$ by an angle $\omega$, as described by a modified (3.3), with $\mathrm{X}_{p}^{(n)^{\prime}}$ replaced by $\overline{\mathrm{X}}_{p}^{(n)}$. We now examine how $\hat{\mathrm{L}}_{i j}^{(n)} g\left(\mathbf{X}^{(n)}\right)$ transforms under that rotation, with $\hat{\mathrm{L}}_{i j}{ }^{(n)}$ defined by (4.1).

We can write, using the inverse of the modified (3.3) to express $\mathbf{X}^{(n)}$ as a function of $\overline{\mathbf{X}}^{(n)}$ and $\omega$,

$$
\begin{aligned}
\left(\frac{\partial}{\partial \omega}\right)_{\overline{\mathbf{X}}_{n}} g\left[\mathbf{X}^{(n)}\left(\overline{\mathbf{X}}^{(n)}, \omega\right)\right]= & \left(\frac{\partial \mathbf{X}_{i}^{(n)}}{\partial \omega}\right)_{\overline{\mathbf{X}}_{n}} \frac{\partial g\left(\mathbf{X}^{(n)}\right)}{\partial \mathbf{X}_{i}^{(n)}} \\
& +\left(\frac{\partial \mathbf{X}_{j}^{(n)}}{\partial \omega}\right)_{\overline{\mathbf{X}}_{n}} \frac{\partial g\left(\mathbf{X}^{(n)}\right)}{\partial \mathbf{X}_{j}^{(n)}} \\
= & \left(-\sin \omega \overline{\mathbf{X}}_{i}^{(n)}-\cos \omega \overline{\mathbf{X}}_{j}^{(n)}\right) \frac{\partial g\left(\mathbf{X}^{(n)}\right)}{\partial \mathbf{X}_{i}^{(n)}} \\
& +\left(\cos \omega \overline{\mathbf{X}}_{i}^{(n)}-\sin \omega \overline{\mathbf{X}}_{j}^{(n)}\right) \frac{\partial g\left(\mathbf{X}^{(n)}\right)}{\partial \mathbf{X}_{j}^{(n)}} \\
= & -\mathbf{X}_{j}^{(n)} \frac{\partial g\left(\mathbf{X}^{(n)}\right)}{\partial \mathbf{X}_{i}^{(n)}}+\mathbf{X}_{i}^{(n)} \frac{\partial g\left(\mathbf{X}^{(n)}\right)}{\partial \mathbf{X}_{j}^{(n)}}
\end{aligned}
$$

In view of (4.1) this gives

$$
\hat{\mathrm{L}}_{i j}^{(n)} g\left(\mathbf{X}^{(n)}\right)=\hat{\mathscr{L}}_{i j}^{(n)} g\left[\mathbf{X}^{(n)}\left(\overline{\mathbf{X}}^{(n)}, \omega\right)\right]
$$

where

$$
\hat{\mathscr{L}}_{i j}^{(n)} \equiv \frac{\hbar}{i}\left(\frac{\partial}{\partial \omega}\right)_{\overline{\mathrm{X}}^{(n)}}
$$

Expressions (4.6) and (4.7) are very important, as they relate the angular momentum operators to derivatives with respect to rotation of the axes angles. It should be stressed that, whereas the $\hat{\mathrm{L}}_{i j}^{(n)}$ operate on the Cartesian coordinates of the particle $\mathrm{P}$, the $\hat{\mathscr{L}}_{i j}^{(n)}$ operate on those rotation angles. It is customary to drop the $\mathrm{g}$ functions on both sides of (4.6) and simply write

$$
\hat{\mathrm{L}}_{i j}^{(n)}=\hat{\mathscr{L}}_{i j}^{(n)}
$$

This expression must be used with great caution, however, since its two sides operate on different variables. It is really a shorthand notation for (4.6).

\subsection{The angular momentum tensor in terms of the Euler angles}

Given a rotation matrix $\mathbf{R}^{(n)}(\boldsymbol{\delta})$ such as (3.9), the tensor transformation relation (4.2), together with (4.6), permits us to express the elements of $\hat{\mathbf{L}}^{(n)}$ and $\overline{\hat{\mathbf{L}}}^{(n)}$ in terms of partial derivatives with respect to the Euler angles contained in $\boldsymbol{\delta}$. 
Indeed, for $n=3$ and the choice (3.6) for $\mathbf{R}^{(3)}(\boldsymbol{\delta})$, we have, from the inverse of (4.2),

$$
\begin{aligned}
\hat{\mathbf{L}}^{(3)}= & \tilde{\mathbf{R}}_{12}^{(3)}\left(\delta^{(1)}\right) \tilde{\mathbf{R}}_{31}^{(3)}\left(\delta^{(2)}\right) \tilde{\mathbf{R}}_{12}^{(3)}\left(\delta^{(3)}\right) \overline{\hat{\mathbf{L}}}^{(3)} \\
& \mathbf{R}_{12}^{(3)}\left(\delta^{(3)}\right) \mathbf{R}_{31}^{(3)}\left(\delta^{(2)}\right) \mathbf{R}_{12}^{(3)}\left(\delta^{(1)}\right)
\end{aligned}
$$

This expression is equivalent to the following set of equations:

$$
\begin{aligned}
& \hat{\mathbf{L}}^{(3)^{\prime \prime}}=\tilde{\mathbf{R}}_{12}^{(3)}\left(\delta^{(3)}\right) \overline{\hat{\mathbf{L}}}^{(3)} \mathbf{R}_{12}^{(3)}\left(\delta^{(3)}\right) \\
& \hat{\mathbf{L}}^{(3)^{\prime}}=\tilde{\mathbf{R}}_{31}^{(3)}\left(\delta^{(2)}\right) \hat{\mathbf{L}}^{(3)^{\prime \prime}} \mathbf{R}_{31}^{(3)}\left(\delta^{(2)}\right) \\
& \hat{\mathbf{L}}^{(3)}=\tilde{\mathbf{R}}_{12}^{(3)}\left(\delta^{(1)}\right) \hat{\mathbf{L}}^{(3)^{\prime}} \mathbf{R}_{12}^{(3)}\left(\delta^{(1)}\right)
\end{aligned}
$$

These 3 equations involve 4 angular momentum tensors, each having 3 independent non-zero elements. Therefore, they establish 9 linear relations between those 12 elements. In addition, however, (4.6) and (4.7) furnish

$$
\begin{aligned}
& \hat{\mathrm{L}}_{12}^{(3)}=\frac{\hbar}{i}\left(\frac{\partial}{\partial \delta^{(1)}}\right)_{\mathbf{X}^{(3)^{\prime}}} \\
& \hat{\mathrm{L}}_{31}^{(3)^{\prime}}=\frac{\hbar}{i}\left(\frac{\partial}{\partial \delta^{(2)}}\right)_{\mathbf{X}^{(3)^{\prime \prime}}} \\
& \hat{\mathrm{L}}_{12}^{(3)^{\prime \prime}}=\frac{\hbar}{i}\left(\frac{\partial}{\partial \delta^{(3)}}\right)_{\overline{\mathbf{X}}^{(3)}}
\end{aligned}
$$

where the $\mathbf{X}$ vectors are related by

$$
\begin{aligned}
& \mathbf{X}^{(3) \prime}=\mathbf{R}_{12}^{(3)}\left(\delta^{(1)}\right) \mathbf{X}^{(3)} \\
& \mathbf{X}^{(3) \prime \prime}=\mathbf{R}_{31}^{(3)}\left(\delta^{(2)}\right) \mathbf{X}^{(3) \prime} \\
& \overline{\mathbf{X}}^{(3)}=\mathbf{R}_{12}^{(3)}\left(\delta^{(3)}\right) \mathbf{X}^{(3) \prime \prime}
\end{aligned}
$$

As a result, the remaining unknown 9 elements can be obtained in terms of the three partial derivatives that appear in the r.h.s. of (4.13) through (4.15). The results for $\hat{\mathrm{L}}_{23}^{(3)}, \hat{\mathrm{L}}_{21}^{(3)}$ and $\hat{\mathrm{L}}_{12}^{(3)}$ are

$$
\begin{aligned}
\hat{\mathrm{L}}_{23}^{(3)}= & \frac{\hbar}{i}\left[-\cos \delta^{(1)} \cot \delta^{(2)}\left(\frac{\partial}{\partial \delta^{(1)}}\right)_{\mathbf{X}^{(3)^{\prime}}}\right. \\
& \left.-\sin \delta^{(1)}\left(\frac{\partial}{\partial \delta^{(2)}}\right)_{\mathbf{X}^{(3)^{\prime \prime}}}+\frac{\cos \delta^{(1)}}{\sin \delta^{(2)}}\left(\frac{\partial}{\partial \delta^{(3)}}\right)_{\overline{\mathbf{x}}^{(3)}}\right] \\
\hat{\mathrm{L}}_{31}^{(3)=} & \frac{\hbar}{i}\left[-\sin \delta^{(1)} \cot \delta^{(2)}\left(\frac{\partial}{\partial \delta^{(1)}}\right)_{\mathbf{X}^{(3)^{\prime}}}\right. \\
& \left.+\cos \delta^{(1)}\left(\frac{\partial}{\partial \delta^{(2)}}\right)_{\mathbf{X}^{(3)^{\prime \prime}}}+\frac{\sin \delta^{(1)}}{\sin \delta^{(2)}}\left(\frac{\partial}{\partial \delta^{(3)}}\right)_{\overline{\mathbf{X}}^{(3)}}\right]
\end{aligned}
$$

$$
\hat{\mathrm{L}}_{12}^{(3)}=\frac{\hbar}{i}\left(\frac{\partial}{\partial \delta^{(1)}}\right)_{\mathbf{X}^{(3)^{\prime}}}
$$

These operator expressions require, in their application to functions, that relations of the type of (4.6) be used, as discussed in connection with (4.7) and (4.8). Eqn (4.19) through (4.21) can be written without the $\mathbf{X}^{(3)}$ subscripts, but they are then implied.

A simpler approach for this 3-dimensional-space case would have been to use the vector transformation relation (4.4) together with (3.6), but that approach would not be generalizable to the $n$-dimensional spaces (with $n>3$ ) of interest in this paper. Setting, as done after (4.4), $\hat{\mathrm{L}}_{23}^{(3)}=\hat{\mathrm{L}}_{x}, \hat{\mathrm{L}}_{31}^{(3)}=\hat{\mathrm{L}}_{y}$ and $\hat{\mathrm{L}}_{12}^{(3)}=\hat{\mathrm{L}}_{z}$, the results of (4.19) through (4.21) agree, of course, with the familiar ones obtained by the vector (or the equivalent infinitesimal rotation) method. ${ }^{43}$

Another case of interest is the 4-dimensional space. Choosing the $\mathbf{R}^{(4)}(\boldsymbol{\delta})$ of (3.7), we can, by this tensor approach, obtain as functions of the Euler angles $\delta^{(p)}(p=1-6)$, the 6 independent elements $\hat{\mathrm{L}}_{12}^{(4)}, \hat{\mathrm{L}}_{31}^{(4)}, \hat{\mathrm{L}}_{14}^{(4)}, \hat{\mathrm{L}}_{23}^{(4)}, \hat{\mathrm{L}}_{42}^{(4)}$ and $\hat{\mathrm{L}}_{34}^{(4)}$ of $\hat{\mathbf{L}}^{(4)}$ that appear in

$$
\hat{\mathbf{L}}^{(4)}=\left(\begin{array}{cccc}
0 & \hat{\mathrm{L}}_{12}^{(4)} & -\hat{\mathrm{L}}_{31}^{(4)} & \hat{\mathrm{L}}_{14}^{(4)} \\
-\hat{\mathrm{L}}_{12}^{(4)} & 0 & \hat{\mathrm{L}}_{23}^{(4)} & -\hat{\mathrm{L}}_{42}^{(4)} \\
\hat{\mathrm{L}}_{31}^{(4)} & -\hat{\mathrm{L}}_{23}^{(4)} & 0 & \hat{\mathrm{L}}_{34}^{(4)} \\
-\hat{\mathrm{L}}_{14}^{(4)} & \hat{\mathrm{L}}_{42}^{(4)} & -\hat{\mathrm{L}}_{34}^{(4)} & 0
\end{array}\right)
$$

The criterion for the selection of these independent elements was the evenness of the permutations of the associated allowed rotations described in Section 3.1.

The explicit expressions for the $\hat{\mathrm{L}}_{i j}^{(4)}$ in terms of the $\partial / \partial \delta^{(p)}$ operators were derived previously by a different but equivalent tensor approach ${ }^{33}$ and will not be repeated here. It should be noted, however, that those $\partial / \partial \delta^{(p)}$ operators should have subscripts that are a straightforward generalization to 4-dimensional space of those indicated in (4.13) through (4.15) for 3-dimensional space. For simplicity, those subscripts have been omitted in the rest of this paper but are implied, and the discussion associated to (4.6) through (4.8) must always be kept in mind. The correspondence between the $\hat{\mathrm{L}}_{\lambda_{k}}$ of ref. 33 and the $\mathrm{L}_{i j}^{(4)}$ is

$$
\begin{array}{lll}
\hat{\mathrm{L}}_{\lambda_{1}}=\hat{\mathrm{L}}_{23}^{(4)} & \hat{\mathrm{L}}_{\lambda_{2}}=\hat{\mathrm{L}}_{31}^{(4)} & \hat{\mathrm{L}}_{\lambda_{3}}=\hat{\mathrm{L}}_{12}^{(4)} \\
\hat{\mathrm{L}}_{\lambda_{4}}=-\hat{\mathrm{L}}_{34}^{(4)} & \hat{\mathrm{L}}_{\lambda_{5}}=\hat{\mathrm{L}}_{42}^{(4)} & \hat{\mathrm{L}}_{\lambda_{6}}=\hat{\mathrm{L}}_{14}^{(4)}
\end{array}
$$

\subsection{The $\hat{\mathbf{L}}^{(n)^{2}}$ operator}

The $\hat{\mathrm{L}}^{(n)^{2}}$ operator of $n$-dimensional space is defined by

$$
\hat{\mathbf{L}}^{(n)^{2}}=\frac{1}{2} \operatorname{tr}\left(\hat{\mathbf{L}}^{(n)^{\mathrm{T}}} \hat{\mathbf{L}}^{(n)}\right)=\frac{1}{2} \operatorname{tr}\left(\hat{\mathbf{L}}^{(n)} \hat{\mathbf{L}}^{(n)^{\mathrm{T}}}\right)
$$

where tr stands for "trace" and the superscript $T$ (as the tilde in (2.2)) stands for "transpose". For 3-dimensional space with $\hat{\mathbf{L}}^{(3)}$ defined by (4.3) we get

$$
\hat{\mathrm{L}}^{(n)^{2}}=\hat{\mathrm{L}}_{23}^{(3)^{2}}+\hat{\mathrm{L}}_{31}^{(3)^{2}}+\hat{\mathrm{L}}_{12}^{(3)^{2}}
$$

This is the sum of the squares of the elements of the 3-dimensional column vector on the r.h.s. of (4.4) and is the usual angular momentum square operator of a point in that 
space. $\hat{\mathbf{L}}^{(n)^{2}}$ transforms under axes rotations as a scalar. Indeed, using (4.24) and remembering that $\hat{\mathrm{L}}^{(n)}$ does not operate on the Euler angles $\boldsymbol{\delta}$ on which $\mathbf{R}^{(n)}$ depends, and that the latter is orthogonal, there results

$$
\begin{aligned}
\overline{\hat{\mathbf{L}}}^{(n)^{\mathrm{T}}} \overline{\hat{\mathbf{L}}}^{(n)} & =\left(\mathbf{R}^{(n)} \hat{\mathbf{L}}^{(n)} \tilde{\mathbf{R}}^{(n)}\right)^{\mathrm{T}}\left(\mathbf{R}^{(n)} \hat{\mathbf{L}}^{(n)} \tilde{\mathbf{R}}^{(n)}\right) \\
& =\left(\mathbf{R}^{(n)} \hat{\mathbf{L}}^{(n)^{\mathrm{T}}} \tilde{\mathbf{R}}^{(n)}\right)\left(\mathbf{R}^{(n)} \hat{\mathbf{L}}^{(n)} \tilde{\mathbf{R}}^{(n)}\right) \\
& =\mathbf{R}^{(n)}\left(\hat{\mathbf{L}}^{(n)^{\mathrm{T}}} \hat{\mathbf{L}}^{(n)}\right) \tilde{\mathbf{R}}^{(n)}
\end{aligned}
$$

This expression proves that the operator $\hat{\mathbf{L}}^{(n)} \hat{\mathbf{L}}^{\mathrm{T}} \hat{}^{(n)}$ transforms under axes rotations as a tensor and is therefore a scalar. Applying the r.h.s. of (4.26) to an arbitrary scalar function $g\left(\mathrm{X}^{(n)}\right)$ we have

$$
\left[\mathbf{R}^{(n)}\left(\hat{\mathbf{L}}^{(n)}{ }^{\mathrm{T}} \hat{\mathbf{L}}^{(n)}\right) \tilde{\mathbf{R}}^{(n)}\right] g\left(\mathbf{X}^{(n)}\right)=\mathbf{R}^{(n)}\left(\hat{\mathbf{L}}^{(n)^{\mathrm{T}}} \hat{\mathbf{L}}^{(n)}\right) g\left(\mathbf{X}^{(n)} \tilde{\mathbf{R}}^{(n)}\right.
$$

Taking the trace of this equation we obtain

$$
\begin{aligned}
& \operatorname{tr}\left\{\left[\mathbf{R}^{(n)}\left(\hat{\mathbf{L}}^{(n)}{ }^{\mathrm{T}} \hat{\mathbf{L}}^{(n)}\right) \tilde{\mathbf{R}}^{(n)}\right] g\left(\mathbf{X}^{(n)}\right)\right\}=\operatorname{tr}\left\{\left[\mathbf{R}^{(n)}\left(\hat{\mathbf{L}}^{(n)^{\mathrm{T}}} \hat{\mathbf{L}}^{(n)}\right) g\left(\mathbf{X}^{(n)}\right) \tilde{\mathbf{R}}^{(n)}\right\}\right. \\
& =\operatorname{tr}\left[\tilde{\mathbf{R}}^{(n)} \mathbf{R}^{(n)}\left(\hat{\mathbf{L}}^{(n)^{\mathrm{T}}} \hat{\mathbf{L}}^{(n)}\right) g\left(\mathbf{X}^{(n)}\right)\right]=\operatorname{tr}\left[\left(\hat{\mathbf{L}}^{(n)^{\mathrm{T}}} \hat{\mathbf{L}}^{(n)}\right) g\left(\mathbf{X}^{(n)}\right)\right]
\end{aligned}
$$

We can now drop the arbitrary function $g\left(\mathrm{X}^{(n)}\right)$ from this expression and obtain the operator relation

$$
\operatorname{tr}\left[\mathbf{R}^{(n)}\left(\hat{\mathbf{L}}^{(n)^{\mathrm{T}}} \hat{\mathbf{L}}^{(n)}\right) \tilde{\mathbf{R}}^{(n)}\right]=\operatorname{tr}\left(\hat{\mathbf{L}}^{(n)^{\mathrm{T}}} \hat{\mathbf{L}}^{(n)}\right)
$$

Taking one half of the trace of both sides of (4.26) and using (4.29) and (4.24) finally furnishes

$$
\overline{\hat{\mathbf{L}}}^{(n)^{2}}=\hat{\mathbf{L}}^{(n)^{2}}
$$

which proves that $\hat{\mathrm{L}}^{(n)^{2}}$ is a scalar operator, Q.E.D.

Great care should be taken in deriving properties of the tensor operator $\hat{\mathrm{L}}^{(n)}$. Because of its skew-symmetry, it is straightforward to prove that it commutes with its transpose, i.e., that

$$
\hat{\mathbf{L}}^{(n)} \hat{\mathbf{L}}^{(n)^{\mathrm{T}}}=\hat{\mathbf{L}}^{(n)} \hat{\mathbf{L}}^{\mathrm{T}(n)}
$$

In spite of this commutativity, $\hat{\mathbf{L}}^{(n)} \hat{\mathbf{L}}^{(n)^{\mathrm{T}}}$ is not a symmetric matrix (as it would be if its elements were functions rather than operators), i.e.,

$$
\left(\hat{\mathbf{L}}^{(n)}\left(\hat{\mathbf{L}}^{(n)^{\mathrm{T}}}\right)^{\mathrm{T}} \neq \hat{\mathbf{L}}^{(n)} \hat{\mathbf{L}}^{(n)^{\mathrm{T}}}\right.
$$

The reason for this lack of symmetry is the non-commutativity among at least some of the elements of $\hat{\mathbf{L}}^{(n)}$. That is so even for the usual $n=3$ case. The safest way to avoid errors in the algebra of these operators is to apply them to an arbitrary function $g\left(\mathbf{X}^{(n)}\right)$, as done in deriving (4.29). Once the corresponding relation involving the $\hat{\mathbf{L}}^{(n)} g\left(\mathbf{X}^{(n)}\right)$ functions is obtained, the $g\left(\mathrm{X}^{(n)}\right)$ can be dropped from both of its sides, thereby giving the desired operator expression.
Replacing (4.19) through (4.21) in (4.25) furnishes, after a considerable amount of algebra, the expression

$$
\begin{aligned}
\hat{\mathrm{L}}^{(3)^{2}=} & -\hbar^{2}\left\{\frac{1}{\sin ^{2} \delta^{(2)}}\left[\left(\frac{\partial^{2}}{\partial \delta^{(1)^{2}}}\right)_{\mathbf{X}^{(3)^{\prime}}}+\left(\frac{\partial^{2}}{\partial \delta^{(3)^{2}}}\right)_{\overline{\mathbf{x}}^{(3)}}\right]\right. \\
& +\frac{1}{\sin \delta^{(2)}}\left(\frac{\partial}{\partial \delta^{(2)}}\right)_{\mathbf{X}^{(3)^{\prime \prime}}} \sin \delta^{(2)}\left(\frac{\partial}{\partial \delta^{(2)}}\right)_{\mathbf{X}^{(3)^{\prime \prime}}} \\
& \left.-2 \frac{\cos \delta^{(2)}}{\sin ^{2} \delta^{(2)}}\left(\frac{\partial}{\partial \delta^{(1)}}\right)_{\mathbf{X}^{(3)^{\prime}}}\left(\frac{\partial}{\partial \delta^{(3)}}\right)_{\overline{\mathbf{x}}^{(3)}}\right\}
\end{aligned}
$$

which agrees with the known result ${ }^{43}$ in which the $\mathbf{X}^{(3)}$ subscripts are omitted but must be implied.

For the $n=4$ case, (4.24) together with (4.22) furnish

$$
\hat{\mathrm{L}}^{(4)^{2}}=\hat{\mathrm{L}}_{12}^{(4)^{2}}+\hat{\mathrm{L}}_{31}^{(4)^{2}}+\hat{\mathrm{L}}_{23}^{(4)^{2}}+\hat{\mathrm{L}}_{14}^{(4)^{2}}+\hat{\mathrm{L}}_{42}^{(4)^{2}}+\hat{\mathrm{L}}_{34}^{(4)^{2}}
$$

Even though the $\hat{\mathrm{L}}_{i j}^{(4)}$ have been obtained previously, ${ }^{33}$ as discussed prior to (4.23), their replacement in (4.34) leads to an excessive amount of trigonometric and partial derivative algebra. Although the first 3 terms in the r.h.s. of (4.34) are the same as for the $n=3$ case, and therefore known, the last 3 involve more than 200 terms before final simplification, and this is not a practical way of obtaining $\hat{\mathrm{L}}^{(4)^{2}}$. We have developed a different approach using matrix methods for deriving this quantity. The expression for $\hat{\mathrm{L}}^{(4)^{2}}$ that results is then modified to permit efficient use of Mathematica. ${ }^{44,45}$ This approach permits a derivation of this quantity in terms of the $\partial / \partial \delta^{(p)}(p=1-6)$ operators, but will not be described in the present paper.

\section{The Wigner rotation operator and function for 3-dimensional and 4-dimensional space}

\subsection{General considerations}

The Wigner rotation function plays a central role in the ROHC theory of reactive scattering. ${ }^{32}$ As an example, it appears in the expressions for the hyperspherical harmonics for tetraatomic systems, which are eigenfunctions of the system's grand-canonical angular momentum operator $\hat{\Lambda}^{2}$ and of a set of other angular momentum operators that commute with it. ${ }^{46}$ For that particular case, it appears twice: once as a function of the 3 Euler angles $\mathbf{a}_{\lambda}$ that rotate the space-fixed frame to the body-fixed principal momentum of inertia frame, and a second time as a function of the 3 internal Euler angles $\boldsymbol{\delta}_{\lambda}$ associated with the rotation matrix $\tilde{\mathbf{R}}^{(3)}\left(\boldsymbol{\delta}_{\lambda}\right)$ of (2.2). In the rest of this section we discuss the definition of the Wigner rotation function and its determination for $(n \geq 3)$-dimensional space.

\subsection{The Wigner rotation operator for $\boldsymbol{n}$-dimensional space}

Although Wigner introduced the concept of a rotation operator for 3 -dimensional space, ${ }^{47}$ the same definition is valid for $n$-dimensional space.

Consider the $n$-dimensional-space coordinates defined at the beginning of Section 3. Let $\psi\left(\mathbf{X}^{(n)}\right)$ be a scalar function of those coordinates. Upon rotation of the $\mathrm{OX}_{1}^{(n)} \mathrm{X}_{2}^{(n)} \ldots \mathrm{X}_{n}^{(n)}$ frame 
to $\mathrm{O} \overline{\mathrm{X}}_{1}^{(n)} \overline{\mathrm{X}}_{2}^{(n)} \ldots \overline{\mathrm{X}}_{n}^{(n)}$, with $\mathrm{P}$ remaining fixed (a so-called "passive" rotation), ${ }^{48,49}$ that function transforms to $\bar{\psi}\left(\overline{\mathbf{X}}^{(n)}\right)$ such that

$$
\bar{\psi}\left(\overline{\mathbf{X}}^{(n)}\right)=\psi\left(\mathbf{X}^{(n)}\right)
$$

i.e., the transformed function $\bar{\psi}$ at the transformed position vector $\overline{\mathbf{X}}^{(n)}$ is equal to the original function $\psi$ at the original position vector $\mathbf{X}^{(n)}$. The Wigner rotation operator $\hat{\mathbf{D}}_{\mathbf{R}^{(n)}}$ is defined by

$$
\hat{\mathbf{D}}_{\mathbf{R}^{(n)}} \psi\left(\overline{\mathbf{X}}^{(n)}\right)=\psi\left(\mathbf{X}^{(n)}\right)
$$

where $\mathbf{R}^{(n)}$ is the coordinate rotation matrix that specifies the rotation of axes being considered. As shown for 3-dimensional space, ${ }^{47,48}$ the $\mathrm{SO}(n)$ groups formed by $\mathbf{R}^{(n)}$ and $\hat{\mathbf{D}}_{\mathbf{R}^{(n)}}$ are isomorphic.

The expression for $\hat{\mathbf{D}}_{\mathbf{R}^{(3)}}$ is given by ${ }^{50}$

$$
\hat{\mathbf{D}}_{\mathbf{R}^{(3)}}=e^{\frac{i}{\hbar} \delta^{(3)}} \hat{\mathbf{L}}_{12}^{(3)} e^{\frac{i}{\hbar} \delta^{(2)}} \hat{\mathbf{L}}_{31}^{(3)} e^{\overbrace{}^{i} \delta^{(1)}} \hat{\mathbf{L}}_{12}^{(3)}
$$

Using an analogous approach it can be shown that for $n=4$ we get

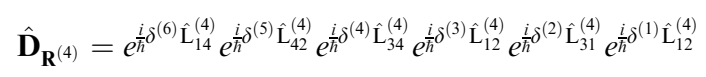

This equation can be easily generalized to the $n$-dimensional case with the choice of $\mathbf{R}^{(n)}(\boldsymbol{\delta})$ given by (3.9).

\subsection{The Wigner rotation function for 3-dimensional space}

Let us consider a system of spinless particles in 3-dimensional space and let the set of all its Cartesian coordinates in the $\mathrm{OX}_{1}^{(3)} \mathrm{X}_{2}^{(3)} \mathrm{X}_{3}^{(3)}$ frame be designated by $\mathbf{r}^{(3)}$. Let furthermore $\psi_{\operatorname{lm}}\left(\mathbf{r}^{(3)}\right)$ be a simultaneous eigenfunction of the square of its total orbital angular momentum operator $\hat{\mathrm{L}}^{(3)^{2}}$ and its $\mathrm{OX}_{3}^{(3)}$ component $\hat{\mathrm{L}}_{23}^{(3)}$. Notice that these symbols are now being used in the context of a system of particles rather than that of the single particle of Section 4. The Wigner rotation matrix of this system in the $l$ representation is, by definition, the square matrix $\mathscr{D}^{(3) l}$ of dimensions $2 l+1$ and whose row $m^{\prime}$ and column $m$ element is ${ }^{41,50}$

$$
\begin{aligned}
\mathscr{D}_{m^{\prime} m}^{(3) l}(\boldsymbol{\delta}) & =\left\langle\psi_{l m^{\prime}}\left(\mathbf{r}^{(3)}\right)\left|\hat{\mathrm{D}}_{\mathbf{R}^{(3)}}\right| \psi_{l m}\left(\mathbf{r}^{(3)}\right)\right\rangle_{\mathbf{r}^{(3)}} \\
& =\left\langle l m^{\prime}\left|\hat{\mathbf{D}}_{\mathbf{R}^{(3)}}\right| l m\right\rangle_{\mathbf{r}^{(3)}} \quad m, m^{\prime}=-l,-l+1, \ldots, l
\end{aligned}
$$

where the subscript $\mathbf{r}^{(3)}$ indicates the integration variable. This element is called a Wigner rotation function and is given explicitly by

$$
\mathscr{D}_{m^{\prime} m}^{(3) l}\left(\delta^{(1)}, \delta^{(2)}, \delta^{(3)}\right)=e^{i m^{\prime} \delta^{(3)}} d_{m^{\prime} m}^{(3) l}\left(\delta^{(2)}\right) e^{i m \delta^{(1)}}
$$

where $d_{m^{\prime} m}^{(3) l}$ is defined as

$$
d_{m^{\prime} m}^{(3) l}\left(\delta^{(2)}\right)=\left\langle l m^{\prime}\left|e^{\frac{i}{\hbar^{(2)}} \hat{L}^{(3)}}\right| \operatorname{lm}\right\rangle_{\mathbf{r}^{(3)}}
$$

and is a known real function of $\delta^{(2)} 50$

We see, from (4.33), that $\hat{\mathrm{L}}^{(n)^{2}}$ commutes with both $\left.\left(\partial / \partial \delta^{(1)}\right) \mathbf{X}^{(3)}\right)^{\prime}$ and $\left(\partial / \partial \delta^{(3)}\right) \overline{\mathbf{X}}^{(3)}$. In addition, the latter commute with each other because $\delta^{(1)}$ and $\delta^{(3)}$ are independent variables. Therefore, those 3 operators form an independent set of commuting operators. It can be shown ${ }^{51}$ that the $\mathscr{D}_{m^{\prime} m}^{(3) l}(\delta)$ functions of (5.6) are simultaneous eigenfunctions of those operators with eigenvalues $l(l+1) \hbar^{2}, m \hbar$ and $m^{\prime} \hbar$ respectively. The corresponding eigenset of partial differential equations is

$$
\begin{aligned}
& -\hbar^{2}\left[\frac{1}{\sin ^{2} \delta^{(2)}}\left(\frac{\partial^{2}}{\partial \delta^{(1)^{2}}}+\frac{\partial^{2}}{\partial \delta^{(3)^{2}}}\right)+\frac{1}{\sin \delta^{(2)}} \frac{\partial}{\partial \delta^{(2)}} \sin \delta^{(2)} \frac{\partial}{\partial \delta^{(2)}}\right. \\
& \left.-2 \frac{\cos \delta^{(2)}}{\sin ^{2} \delta^{(2)}} \frac{\partial^{2}}{\partial \delta^{(1)} \partial \delta^{(3)}}\right] \mathscr{D}_{m^{\prime} m}^{(3) l}\left(\delta^{(1)}, \delta^{(2)}, \delta^{(3)}\right) \\
& =l(l+1) \hbar^{2} \mathscr{D}_{m^{\prime} m}^{(3) l}\left(\delta^{(1)}, \delta^{(2)}, \delta^{(3)}\right)
\end{aligned}
$$

$$
\begin{aligned}
& \frac{\hbar}{i} \frac{\partial}{\partial \delta^{(1)}} \mathscr{D}_{m^{\prime} m}^{(3) l}\left(\delta^{(1)}, \delta^{(2)}, \delta^{(3)}\right)=m \hbar \mathscr{D}_{m^{\prime} m}^{(3) l}\left(\delta^{(1)}, \delta^{(2)}, \delta^{(3)}\right) \\
& \frac{\hbar}{i} \frac{\partial}{\partial \delta^{(3)}} \mathscr{D}_{m^{\prime} m}^{(3) l}\left(\delta^{(1)}, \delta^{(2)}, \delta^{(3)}\right)=m^{\prime} \hbar \mathscr{D}_{m^{\prime} m}^{(3) l}\left(\delta^{(1)}, \delta^{(2)}, \delta^{(3)}\right)
\end{aligned}
$$

Together with the normalization condition

$$
\mathscr{D}_{m^{\prime} m}^{(3) l}(0,0,0)=1
$$

these equations are equivalent to (5.5) or (5.6). Replacement of (5.6) in (5.8) shows that the $d_{m^{\prime} m}^{(3) l}$ function is a solution of the ordinary second order differential equation ${ }^{51}$

$$
\begin{aligned}
& -\hbar^{2}\left[\frac{1}{\sin \delta^{(2)}} \frac{\mathrm{d}}{\mathrm{d} \delta^{(2)}} \sin \delta^{(2)} \frac{\mathrm{d}}{\mathrm{d} \delta^{(2)}}\right. \\
& \left.-\frac{m^{2}+m^{\prime 2}-2 m m^{\prime} \cos \delta^{(2)}}{\sin ^{2} \delta^{(2)}}\right] \mathrm{d}_{m^{\prime} m}^{(3) l}\left(\delta^{(2)}\right)=l(l+1) \hbar^{2} \mathrm{~d}_{m^{\prime} m}^{(3) l}\left(\delta^{(2)}\right)
\end{aligned}
$$

and is simply related to a Jacobi polynomial of $\cos \delta^{(2)}$.

In the partial derivatives that appear in (5.8) through (5.10) no $\mathbf{X}^{(3)}$ or $\mathbf{r}^{(3)}$ subscripts are needed because $\mathscr{D}_{m^{\prime} m}^{(3) l}$ is independent of the Cartesian coordinates of the point (or points) comprising the system and depends only on the Euler angles $\delta^{(1)}, \delta^{(2)}, \delta^{(3)}$. Those equations make the $\mathscr{D}_{m^{\prime} m}^{(3) l}$ useful basis functions for expanding the tetraatomic system's wave functions. ${ }^{32,46}$ The fact that they stem from the matrix representation of the operator (5.3) is not central for this application.

\subsection{The Wigner rotation function for 4-dimensional space}

We now define a set of functions of $\boldsymbol{\delta} \equiv\left(\delta^{(1)}, \delta^{(2)}, \ldots, \delta^{(6)}\right)$ that has properties analogous to those of (5.8) through (5.11), i.e. that are simultaneous eigenfunctions of an appropriate set of angular momentum operators in 4-dimensional space. Rather than starting with (5.4) and seeking its representation in an appropriate basis set, we search instead for a set of 6 linearlyindependent commuting angular momentum operators in the space spanned by $\boldsymbol{\delta}$ and whose simultaneous eigenfunctions will be the functions of interest. This approach yields directly a basis set useful for expanding the wave functions of pentaatomic systems. ${ }^{33}$

To that effect, let us consider the operators $\hat{\mathrm{L}}_{i j}^{(4)}$ of (4.22) and the associated $\hat{\mathrm{L}}^{(4)^{2}}$ of (4.34). In addition, let us define the operator $\hat{\mathrm{L}}^{t(4)^{2}}$ by

$$
\hat{\mathbf{L}}^{t(4)^{2}}=\hat{\mathbf{L}}_{12}^{(4)^{2}}+\hat{\mathbf{L}}_{23}^{(4)^{2}}+\hat{\mathbf{L}}_{31}^{(4)^{2}}
$$


in terms of which $\hat{\mathrm{L}}^{(4)^{2}}$ can be written as

$$
\hat{\mathbf{L}}^{(4)^{2}}=\hat{\mathbf{L}}^{t(4)^{2}}+\hat{\mathbf{L}}_{14}^{(4)^{2}}+\hat{\mathbf{L}}_{42}^{(4)^{2}}+\hat{\mathbf{L}}_{34}^{(4)^{2}}
$$

From the definition (4.1) of the $\hat{\mathrm{L}}_{i j}^{(4)}$ it can be proven that the 3 independent operators $\hat{\mathrm{L}}^{(4)^{2}}, \hat{\mathrm{L}}^{t(4)^{2}}$ and $\hat{\mathrm{L}}_{12}^{(4)}$ commute pair-wise, and that no more that 3 such independent commuting operators can be constructed from the elements of (4.22).

Let us also consider the frames generated from $\mathrm{OX}_{1}^{(4)} \mathrm{X}_{2}^{(4)} \mathrm{X}_{3}^{(4)} \mathrm{X}_{4}^{(4)}$ by successive rotations by the $\mathbf{R}_{i j}^{(4)}$ matrices that appear in (3.7):

$$
\begin{aligned}
& \mathbf{R}_{12}^{(4)}\left(\delta^{(1)}\right) \mathrm{OX}_{1}^{(4)} \mathrm{X}_{2}^{(4)} \mathrm{X}_{3}^{(4)} \mathrm{X}_{1}^{(4)} \rightarrow \mathrm{OX}_{1^{\prime}}^{(4)} \mathrm{X}_{2^{\prime}}^{(4)} \mathrm{X}_{3^{\prime}}^{(4)} \mathrm{X}_{4^{\prime}}^{(4)} \\
& \mathbf{R}_{31}^{(4)}\left(\delta^{(2)}\right) \mathrm{OX}_{1^{\prime}}^{(4)} \mathrm{X}_{2^{\prime}}^{(4)} \mathrm{X}_{3^{\prime}}^{(4)} \mathrm{X}_{4^{\prime}}^{(4)} \rightarrow \mathrm{OX}_{1^{\prime \prime}}^{(4)} \mathrm{X}_{2^{\prime \prime}}^{(4)} \mathrm{X}_{3^{\prime \prime}}^{(4)} \mathrm{X}_{4^{\prime \prime}}^{(4)} \\
& \mathbf{R}_{12}^{(4)}\left(\delta^{(3)}\right) \mathrm{OX}_{1^{\prime \prime}}^{(4)} \mathrm{X}_{2^{\prime \prime}}^{(4)} \mathrm{X}_{3^{\prime \prime}}^{(4)} \mathrm{X}_{4^{\prime \prime}}^{(4)} \rightarrow \mathrm{OX}_{1^{\prime \prime \prime}}^{(4)} \mathrm{X}_{2^{\prime \prime \prime}}^{(4)} \mathrm{X}_{3^{\prime \prime \prime}}^{(4)} \mathrm{X}_{4^{\prime \prime \prime}}^{(4)} \\
& \mathbf{R}_{34}^{(4)}\left(\delta^{(4)}\right) \mathrm{OX}_{1^{\prime \prime \prime}}^{(4)} \mathrm{X}_{2^{\prime \prime \prime}}^{(4)} \mathrm{X}_{3^{\prime \prime \prime}}^{(4)} \mathrm{X}_{4^{\prime \prime \prime}}^{(4)} \rightarrow \mathrm{OX}_{1^{\mathrm{IV}}}^{(4)} \mathrm{X}_{2^{\mathrm{IV}}}^{(4)} \mathrm{X}_{3^{\mathrm{IV}}}^{(4)} \mathrm{X}_{4^{\mathrm{IV}}}^{(4)} \\
& \mathbf{R}_{42}^{(4)}\left(\delta^{(5)}\right) \mathrm{OX}_{1^{\mathrm{IV}}}^{(4)} \mathrm{X}_{2^{\mathrm{IV}}}^{(4)} \mathrm{X}_{3^{\mathrm{IV}}}^{(4)} \mathrm{X}_{4^{\mathrm{IV}}}^{(4)} \rightarrow \mathrm{OX}_{1^{\mathrm{V}}}^{(4)} \mathrm{X}_{2^{\mathrm{V}}}^{(4)} \mathrm{X}_{3^{\mathrm{V}}}^{(4)} \mathrm{X}_{4^{\mathrm{V}}}^{(4)} \\
& \mathbf{R}_{14}^{(4)}\left(\delta^{(6)}\right) \mathrm{OX}_{1^{\mathrm{V}}}^{(4)} \mathrm{X}_{2^{\mathrm{V}}}^{(4)} \mathrm{X}_{3^{\mathrm{V}}}^{(4)} \mathrm{X}_{4^{\mathrm{V}}}^{(4)} \rightarrow \mathrm{OX}_{1^{\mathrm{VI}}}^{(4)} \mathrm{X}_{2^{\mathrm{VI}}}^{(4)} \mathrm{X}_{3^{\mathrm{VI}}}^{(4)} \mathrm{X}_{4^{\mathrm{VI}}}^{(4)}
\end{aligned}
$$

We now select the following 3 elements of the tensors in some of these frames:

$$
\begin{aligned}
& \hat{\mathrm{L}}_{1^{\prime \prime 2} 2^{\prime \prime}}^{(4)}=\hat{\mathrm{L}}_{1^{\prime \prime \prime} 2^{\prime \prime \prime}}^{(4)}=\frac{\hbar}{i} \frac{\partial}{\partial \delta^{(3)}} \\
& \hat{\mathrm{L}}_{3^{\prime \prime \prime} 4^{\prime \prime \prime}}^{(4)}=\hat{\mathrm{L}}_{3^{\mathrm{IV}} 4^{\mathrm{IV}}}^{(4)}=\frac{\hbar}{i} \frac{\partial}{\partial \delta^{(4)}} \\
& \hat{\mathrm{L}}_{1^{\mathrm{V}} 4^{\mathrm{V}}}^{(4)}=\hat{\mathrm{L}}_{1^{\mathrm{VI}} 4^{\mathrm{VI}}}^{(4)}=\frac{\hbar}{i} \frac{\partial}{\partial \delta^{(6)}}
\end{aligned}
$$

The operators $\hat{\mathrm{L}}_{1^{\prime \prime} 2^{\prime \prime}}^{(4)}, \hat{\mathrm{L}}_{3^{\prime \prime \prime} 4^{\prime \prime \prime}}^{(4)}$ and $\hat{\mathrm{L}}_{1^{\mathrm{VI}} 4^{\mathrm{VI}}}^{(4)}$ commute with each other because they operate on the independent variables $\delta^{(3)}$, $\delta^{(4)}, \delta^{(6)}$. In addition, they commute with $\hat{\mathrm{L}}^{(4)^{2}}, \hat{\mathrm{L}}^{t(4)^{2}}$ and $\hat{\mathrm{L}}_{12}^{(4)}$. The last two are given by (4.33) and (4.21) respectively (with the subscripts omitted) whereas the determination of $\hat{L}^{(4)^{2}}$ was discussed after (4.34). This is the set of 6 commuting operators of interest, and their simultaneous eigenfunctions $\mathscr{D}_{m_{3} m_{4} m_{6}}^{(4) l \mu m_{1}}(\boldsymbol{\delta})$ satisfy the partial differential equations

$$
\begin{aligned}
& \hat{\mathrm{L}}_{2}^{(4)^{2}} \mathscr{D}_{m_{3} m_{4} m_{6}}^{(4) l \mu m_{1}}(\boldsymbol{\delta})=l(l+2) \hbar^{(2)} \mathscr{D}_{m_{3} m_{4} m_{6}}^{(4) l \mu m_{1}}(\boldsymbol{\delta}) \\
& \hat{\mathrm{L}}^{t(4)^{2}} \mathscr{D}_{m_{3} m_{4} m_{6}}^{(4) l \mu m_{1}}(\boldsymbol{\delta})=\mu(\mu+1) \hbar^{2} \mathscr{D}_{m_{3} m_{4} m_{6}}^{(4) l \mu m_{1}}(\boldsymbol{\delta}) \\
& \hat{\mathrm{L}}_{12}^{(4)} \mathscr{D}_{m_{3} m_{4} m_{6}}^{(4) l \mu m_{1}}(\boldsymbol{\delta})=m_{1} \hbar \mathscr{D}_{m_{3} m_{4} m_{6}}^{(4) l \mu m_{1}}(\boldsymbol{\delta}) \\
& \hat{\mathrm{L}}_{1^{\prime \prime} 2^{\prime \prime}}^{(4)} \mathscr{D}_{m_{3} m_{4} m_{6}}^{(4) l \mu m_{1}}(\boldsymbol{\delta})=m_{3} \hbar \mathscr{D}_{m_{3} m_{4} m_{6}}^{(4) l m_{1}}(\boldsymbol{\delta}) \\
& \hat{\mathrm{L}}_{3^{\prime \prime \prime} 4^{\prime \prime \prime}}^{(4)} \mathscr{D}_{m_{3} m_{4} m_{6}}^{(4) l \mu m_{1}}(\boldsymbol{\delta})=m_{4} \hbar \mathscr{D}_{m_{3} m_{4} m_{6}}^{(4) l \mu m_{1}}(\boldsymbol{\delta}) \\
& \hat{\mathrm{L}}_{1^{\mathrm{VI}} 4^{\mathrm{VI}}}^{(4)} \mathscr{D}_{m_{3} m_{4} m_{6}}^{(4) l \mu m_{1}}(\boldsymbol{\delta})=m_{6} \hbar \mathscr{D}_{m_{3} m_{4} m_{6}}^{(4) l \mu m_{1}}(\boldsymbol{\delta})
\end{aligned}
$$

The quantum numbers $l, \mu, m_{1}, m_{3}, m_{4}$ and $m_{6}$ are all integers and their ranges are given by ${ }^{52}$

$$
\begin{aligned}
l & =0,1,2, \ldots \\
\mu & =0,1,2 \ldots, l \\
m_{1} & =-\mu,-\mu+1, \ldots, \mu \\
m_{j} & =-l,-l+1, \ldots, l \quad j=3,4,6
\end{aligned}
$$

The superscripts $l, \mu, m_{1}$ and subscripts $m_{3}, m_{4}, m_{6}$ in the $\mathscr{D}^{(4)}(\delta)$ functions are identifying indices that have not been organized so as to define the rows and columns of a square matrix.

It should be noted that (5.26) through (5.29) are very simple first order differential equations whose solutions are $e^{i m_{j} \delta^{(j)}}$, with $j=1,3,4$ and 6 respectively. Eqn (5.25) is very similar to the (5.8) second order partial differential equation satisfied by the $\mathscr{D}_{m^{\prime} m}^{(3) l}\left(\delta^{(1)}, \delta^{(2)}, \delta^{(3)}\right)$ function. Finally, (5.24) is also a second order partial differential equation in the 6 variables $\delta^{(p)}(p=1-6)$. However, when the solutions of (5.25) through (5.29) are factored out from $\mathscr{D}_{m_{3} m_{4} m_{6}}^{(4) l \mu m_{1}}$, the remaining coefficient function depends on $\delta^{(5)}$ only, and satisfies an ordinary second order differential equation similar to (5.12) that is susceptible to analytical solution. In this manner the $\mathscr{D}_{m_{3} m_{4} m_{6}}^{(4) l m_{1}}$ can be obtained explicitly, but this explicit expression will be the subject of a separate publication. It seems that this partial differential equation approach for obtaining the $\mathscr{D}$ Wigner rotation function for 3- and 4-dimensional space is extendable to higher dimensions.

\section{Summary and conclusions}

In this paper, rotations in $n$-dimensional space and the associated tensor angular momentum operator and Euler angles were discussed. That operator was expressed in terms of those angles using the transformation properties of second order tensors under axes rotations. The concept of Wigner rotation operator and function was generalized to $n$-dimensional space and it was shown that the Wigner rotation function for 4-dimensional space is obtained by solving an appropriate set of eigenfunction-eigenvalue partial differential equations associated with six commuting angular momentum operators. The resulting solutions constitute a useful basis for expanding the hyperspherical harmonics for pentaatomic systems, in analogy to tetraatomic systems. ${ }^{32,45}$ Furthermore, this approach is generalizable to higher dimensional spaces.

The $F$ hyperspherical harmonics ${ }^{32,46}$ for pentaatomic systems depends on 11 angles: the 3 Euler angles that rotate the space-fixed axes to the principal moment of inertia bodyfixed axes, the 6 internal Euler angles of a 4-dimensional space, and two principal moment of inertia angles. The basis set in the first 3 is the usual 3-dimensional Wigner rotation function, and in the next 6 it is the 4-dimensional space Wigner rotation function discussed in this paper. For the last two angles it is the $G$ hyperspherical harmonics set. The latter functions are not presently known, but they may be determined by an extension of the method used previously to obtain them for triatomic ${ }^{34}$ and tetraatomic ${ }^{46}$ systems. Once they are obtained, all of the analytical tools needed for calculating accurate 
state-to-state differential and integral cross sections for pentaatomic reactions (such as $\mathrm{H}_{2}+\mathrm{H}_{3}^{+}$and its isotopomers, which are important for interstellar processes ${ }^{53}$ ) will be available. It is estimated that such calculations can be performed in about $10 \mathrm{~h}$ on a 10 teraflop computer.

It may also be possible to obtain the Wigner rotation function for 5-dimensional space by the methods described. Together with the determination of the corresponding $G$ harmonics, this may make the ab initio calculation of stateto-state cross sections for hexaatomic reactions feasible in the foreseeable future, using petaflop computers. Finally, the ROHC approach is also applicable to the calculation of bound-state energies of small polyatomic systems, as well as to the study of bound states of small nuclei and of their reactions. Reactive scattering calculations using ROHC can be performed by solving the corresponding time-independent or time-dependent Schrödinger equation.

\section{Acknowledgements}

The author wishes to express his appreciation to Prof. Raphael D. Levine, who some 35 years ago first called his attention to early work in hyperspherical coordinates.

\section{References}

1 W. Hu and G. C. Schatz, J. Chem. Phys., 2006, 125, 132301.

2 D. H. Zhang, M. Yang, S.-Y. Lee and M. A. Collins, in Modern Trends in Chemical Reaction Dynamics, ed. X. Yang and K. Liu, World Scientific, New Jersey, 2004, pp. 409-464.

3 S. C. Althorpe and D. C. Clary, Annu. Rev. Phys. Chem., 2003, 54, 493-529.

4 D. C. Clary, Science, 1998, 279, 1879.

5 J. M. Bowman and G. C. Schatz, Annu. Rev. Phys. Chem., 1995, 46, 169-195.

6 M. T. Cvitaš and S. C. Althorpe, Phys. Scr., 2009, 80, 048115.

7 M. Launay and M. le Dourneuf, Chem. Phys. Lett., 1989, 163, 178.

8 M. Launay, Theor. Chim. Acta, 1991, 79, 183.

9 S. Branchett, S. B. Padkjaer and J. M. Launay, Chem. Phys. Lett., 1993, 208, 523.

10 Y.-S. M. Wu, A. Kuppermann and B. Lepetit, Chem. Phys. Lett., 1991, 196, 319.

11 M. P. Miranda, D. C. Clary, J. F. Castillo and D. E. Manolopoulos, J. Chem. Phys., 1998, 108, 3142.

12 D. Skouteris, J. F. Castillo and D. E. Manolopoulos, Comput. Phys. Commun., 2000, 133, 128.

13 S. D. Chao and R. T. Skodje, J. Chem. Phys., 2000, 113, 3487.

14 F. Fernandez-Alonso, B. D. Bean, R. N. Zare, F. J. Aoiz, L. Bañares and J. F. Castilo, J. Chem. Phys., 2001, 115, 4534.

15 B. Kendrick, J. Chem. Phys., 2001, 114, 8796.

16 V. Aquilanti, S. Cavalli, D. de Fazio, A. Volpi, A. Aguilar, X. Giménez and J. M. Lucas, Phys. Chem. Chem. Phys., 2002, 4, 401.

17 D. de Fazio, V. Aquilanti, S. Cavalli, A. Aguilar and J. M. Lucas, J. Chem. Phys., 2008, 129, 064303.

18 B. Lepetit, R. Abrol and A. Kuppermann, Phys. Rev. A: At., Mol., Opt. Phys., 2007, 76, 040702.
19 F. Bouakline, B. Lepetit, S. C. Althorpe and A. Kuppermann, in The Jahn-Teller Effect: Fundamentals and Implications for Physics and Chemistry, ed. H. Köppel, D. R. Yarkony and H. Barentzen, Springer-Verlag, New York, 2009, pp. 201-237.

20 G. A. Parker and R. T. Pack, J. Chem. Phys., 1993, 98, 6883.

21 L. Bañares, F. J. Aoiz, B. Honvault and B. Bussery-Honvault, J. Chem. Phys., 2003, 118, 565.

22 S. A. Harich, D. Dai, C. C. Wang, X. Yang, S. D. Chao and R. T. Skodje, Nature, 2002, 419, 281.

23 M. Qiu, Z. Ren, D. Che, S. A. Harich, X. Wang, X. Yang, C. Xu, D. Xie, M. Gustafsson, R. T. Skodje, Z. Sun and D. H. Zhang, Science, 2006, 311, 1440.

24 S. K. Pogrebnya, J. Echave and D. C. Clary, J. Chem. Phys., 1997, 107, 8975 .

25 S. K. Pogrebnya, J. Palma, D. C. Clary and J. Echave, Phys. Chem. Chem. Phys., 2000, 2, 693.

26 B. Lepetit, Z. Peng and A. Kuppermann, Chem. Phys. Lett., 1990, 166, 572.

27 L. Velilla, B. Lepetit, A. Aguado, J. A. Beswick and M. Panagua, J. Chem. Phys., 2008, 129, 084307.

28 R. G. Littlejohn, K. A. Mitchell and V. Aquilanti, Phys. Chem. Chem. Phys., 1999, 1, 1259.

29 A. Kuppermann, in Advances in Molecular Vibrations and Collision Dynamics, ed. J. M. Bowman, JAI Press, Greenwich, Connecticut, 1994, vol. 2B, pp. 117-186.

30 A. Kuppermann, J. Phys. Chem., 1996, 100, 2621, 11202.

31 A. Kuppermann, J. Phys. Chem., 1997, 101, 6368.

32 A. Kuppermann, Isr. J. Chem., 2003, 43, 229.

33 A. Kuppermann, J. Phys. Chem. A, 2009, 113, 4518.

34 D. Wang and A. Kuppermann, J. Phys. Chem. A, 2009, 113, 15384

35 G. H. Golub and C. F. Van Loan, Matrix Computations, Johns Hopkins University Press, Baltimore, Maryland, 1933, chap. 12, Section 8.3 .

36 W. H. Press, B. P. Flannery, S. A. Teukolsky and W. T. Veterling, Numerical Recipes, Cambridge University Press, Cambridge, 1986, Section 2.9.

37 Y. Ohrn and J. Linderberg, Mol. Phys., 1983, 49, 53.

38 W. Zickendraht, J. Math. Phys., 1969, 10, 30.

39 V. Aquilanti and S. Cavalli, J. Chem. Soc., Faraday Trans., 1997, 93, 801.

40 V. Aquilanti, A. Lombardi and E. Yurtsever, Phys. Chem. Chem. Phys., 2002, 4, 5040.

41 E. P. Wigner, Group Theory, Academic Press, New York, 1959, chap. 15 .

42 H. Goldstein, Classical Mechanics, Addison-Wesley, Reading, Massachusetts, 1959, p. 130.

43 A. R. Edmonds, Angular Momentum in Quantum Mechanics, Princeton University Press, New Jersey, 1968, p. 13.

44 S. Wolfram, Mathematica, 2nd edn, Addison-Wesley, Reading, Massachusetts, 1992.

45 D. Wang and A. Kuppermann, Int. J. Quantum Chem., 2006, 106, 152.

46 D. Wang and A. Kuppermann, J. Chem. Phys., 2001, 115, 9184.

47 ref. 41, Chap. 11.

48 M. Bouten, Physica, 1969, 42, 572.

49 R. N. Zare, Angular Momentum, John Wiley \& Sons, New York, 1988, pp. 104, 107.

50 Ref. 43 , pp. $55-57$

51 Ref. 43, p. 65.

52 J. Avery, Hyperspherical Harmonics, Kluwer, Dordrecht, the Netherlands, 1989 , pp. 38-44.

53 D. Gerlich, E. Herbst and E. Roueff, Planet. Space Sci., 2002, 50, 1275 